\title{
Notfall-Babys immer anschnallen!
}

\author{
Beim Notfall-Transport von Neuge- \\ borenen, Frühgeborenen und Säug- \\ lingen, kommt meist ein Inkubator \\ im Rettungswagen zum Einsatz. \\ Dies ist jedoch nicht ungefährlich, \\ denn die kleinen Patienten werden \\ bei einer Vollbremsung massiv ge- \\ gen die Scheibe gedrückt.
}

In einer Untersuchung mit verschiedenen Fahrzeugausstattungen und insgesamt 60 Testfahrten zeigte sich, dass der Säuglingsdummy „Willi“ bei jeder Vollbremsung gegen das Inkubatorglas in Fahrtrichtung gedrückt wurde. Dabei traten erhebliche Beschleunigungskräfte auf, schreiben Dr. Gernot Rücker und Kollegen vom Uniklinkium Rostock in „Notfall- und Rettungsmedizin“.

\section{Quertransport von Vorteil}

Die Ergebnisse der Vollbremsungstests ergaben, dass die Verzögerungskräfte im konventionellen Rettungswagen mit Inku- bator mit Längstransport im Vergleich zum Baby-Notarztwagen-Modell (Quertransport, Baby-NAW 1) deutlich höher waren. Der Quertransport ist daher von Vorteil, da insgesamt weniger Kräfte auf den Säugling wirken. Die Autoren fordern

außerdem, die kleinen Patienten in jedem Fall mit Rückhaltesystemen zu schützen, um im Falle einer Kollision oder Vollbremsung Schäden zu vermeiden.

Rücker G et al, Notfall Rettungsmed 2012, 15:690

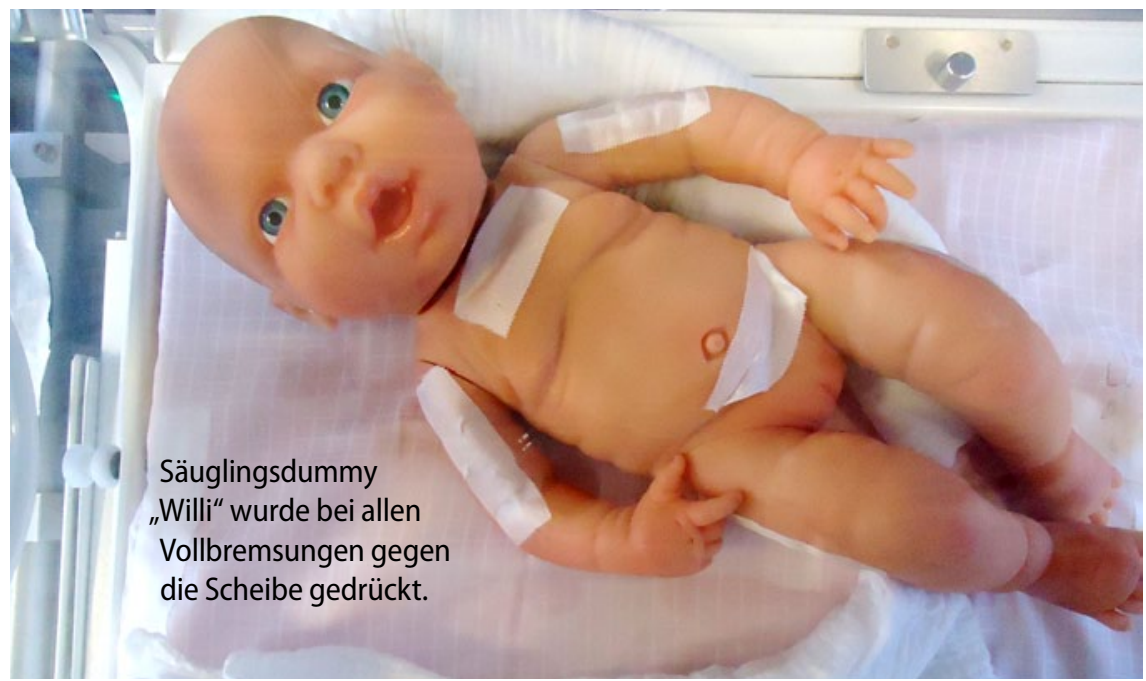

höhere Gewichtszunahme als Frauen, die Kinder mit Geburtsgewichten von 25003916 g gebären. Der Mittelwert der Gewichtszunahme im Untersuchungskollektiv betrug $12,4 \mathrm{~kg}$, die Standardabweichung 8,4. Eine überdurchschnittliche Gewichtszunahme $(\geq+s)$ liegt ab $20,8 \mathrm{~kg}$ vor. Dies trifft auf 15,3\% der Population zu. Frauen mit überdurchschnittlicher Gewichtszunahme unterscheiden sich präkonzeptionell weder im Gewicht noch in der Körpergröße noch im BMI von den anderen Schwangeren.

Bis vor dem Geburtstermin erhöhte sich das Gewicht im Mittel um 25 kg gegenüber $14 \mathrm{~kg}$ in der Vergleichsgruppe. Zum Zeitpunkt der Zweitbefragung (ca. 20 Monate nach Geburt) betrug die Gewichtsdifferenz zum präkonzeptionellen Gewicht in der Vergleichsgruppe nur 1,3 kg vs. 5,4 kg in der Gruppe mit hoher Gewichtszunahme.

Frauen mit überdurchschnittlicher Gewichtszunahme hatten damit auch 20 Monate nach der Geburt gegenüber dem präkonzeptionellen Gewicht ein höheres Gewicht, während in der Vergleichsgrup- pe kein signifikanter Unterschied zum „Ausgangsgewicht“ besteht. Frauen mit überdurchschnittlicher Gewichtszunahme haben mit $18,8 \%$ zu $9,1 \%$ doppelt so häufig übergewichtige Kinder ( $\geq 4000 \mathrm{~g}$ ).

\section{Was zeigt der Gruppenvergleich?}

Vergleicht man die Gruppen nach soziodemografischen Faktoren und der Lebensweise, so zeigt sich, dass Schwangere, die überdurchschnittlich an Gewicht zunehmen, signifikant jünger sind, häufiger eine einfache Schulbildung und ein geringeres Haushaltseinkommen haben und häufiger der unteren sozialen Schicht angehören, bei Diagnose der Schwangerschaft und in der Schwangerschaft häufiger rauchen, häufiger keinen Sport betreiben, sich hinsichtlich des Auftretens von Komplikationen im Verlauf der Schwangerschaft jedoch nicht unterscheiden. Zum Zeitpunkt der Erstbefragung zeigten sich keine signifikanten Unterschiede in der Energieaufnahme, jedoch eine in starker Tendenz höhere Fettaufnahme.

Derzeit können auf wissenschaftlich gesicherter Grundlage keine belastbaren
Empfehlungen für eine angemessene Gewichtszunahme in der Schwangerschaft gegeben werden.

Ob bei der Frage, wie viel Gewichtszunahme nötig und welche gefährlich ist, die IOM-Empfehlungen hilfreich sein können, darf v. a. aus zwei Gründen bezweifelt werden. Erstens berücksichtigen diese die Körpergrößenklassen nicht. Zweitens überschreiten in unserem Datensatz insgesamt 43\% der Schwangeren diese Zunahmeempfehlungen und nur $23 \%$ sind im „Normbereich“.

Für den niedergelassenen Arzt in der Schwangerenvorsorge heißt das, weiterhin regelmäßig die Gewichtszunahme in der Schwangerschaft zu kontrollieren, die aber mit den von Voigt u. a. erarbeiteten Normwertkurven [9] nach Gewicht und Alter abgeglichen werden sollte. Es besteht aber zweifellos hoher Forschungsbedarf zur Frage nach der angemessenen Gewichtszunahme in der Schwangerschaft.

Die Langfassung mit Tabellen und Literatur finden Sie unter: www.springermedizin.de/2314330 